\title{
Re-framing HCI through Local and Indigenous Perspectives
}

\author{
Jose Abdelnour-Nocera ${ }^{1}$, Masaaki Kurosu², Torkil Clemmensen ${ }^{3}$, Nic Bidwell ${ }^{4}$, \\ Ravi Vatrapu ${ }^{3}$, Heike Winschiers-Theophilus ${ }^{5}$, Vanessa Evers ${ }^{6}$, \\ Rüdiger Heimgärtner ${ }^{7}$, and Alvin $\mathrm{Yeo}^{8}$ \\ ${ }^{1}$ University of West London \\ ${ }^{2}$ The Open University of Japan \\ ${ }^{3}$ Copenhagen Business School \\ ${ }^{4}$ CSIR-Meraka \& Nelson Mandela Metropolitan University \\ ${ }^{5}$ Polytechnic of Namibia \\ ${ }^{6}$ University of Amsterdam \\ ${ }^{7}$ Intercultural User Interface Consulting Germany \\ ${ }^{8}$ Universiti Malaysia Sarawak \\ Jose. Abdelnour-Nocera@uwl.ac.uk \\ masaakikurosu@spa.nifty.com, tc.inf@cbs.dk, \\ nic.bidwell@gmail.com, rv.caict@cbs.dk, \\ heikew@polytechnic.edu.na, evers@uva.nl, \\ ruediger.heimgaertner@iuic.de, awyeo@acm.org
}

\begin{abstract}
This one-day workshop aims to present different local and indigenous perspectives from all over the world in order to lead into an international dialogue on re-framing concepts and models in HCI/Interaction Design. The target audience is HCI researchers and practitioners who have experience with working with culture and HCI. The expected outcome of the workshop is a) network building among the participants, b) a shortlist of papers that can be basis for a proposal for a special issue of the UAIS journal, and c) identify opportunities to develop a funded network or research proposal.
\end{abstract}

Keywords: Indigenous HCI, HCI theory and methodology, localization, globalization, cultural usability.

\section{Objective and Theme}

One of the current challenges for HCI as a discipline is addressing the tensions created between local cultures and the assumptions, priorities and values embedded in the HCI as a discipline. The objective of this workshop is to further our understanding of these issues and lead to practical recommendations for people researching and implementing HCI from an explicit indigenous perspective. Translating local knowledge into valid and useful HCI tools is not a simple problem, but one that requires re-defining and renegotiating disciplinary boundaries (and connections) and the subject and object of the interaction design. Focusing on local or indigenous awareness and practices in design pushes the envelope in a very exciting way. For instance, the democratic values of equal participation driving user-centred design are not necessarily shared by local communities which prioritize respecting the views of their leaders. Addressing these 
gaps requires a fresh look at how diverse disciplines and professions explore and conceptualize the relation between users, designers and other stakeholders. While the global HCI community has well-defined conceptual and methodological frameworks, there is little research about how local HCI professionals experience, adapt and implement this knowledge, nor how to locate HCI so that it is locally accountable [1]. To progress this research we must start by better understanding relationships between HCI concepts and methods and their meanings to local and indigenous groups. Universal perspectives on HCI like ethnology and ethnography, e.g., technomethodology [2], and national culture models [3] and activity theory [4] have all had an impact in the design of interactive systems for culturally different users, but the potential contribution of explicitly local or indigenous perspectives, approaches and experiences with HCI, see e.g., [5], have not become so clear and uniform. Furthermore, the idea of what constitutes a useful and usable system in different cultural contexts remains partially explored at the very least. In addition, a simple localization, i.e. the adaptation of products and systems developed in advanced countries to developing countries, may take the form of cultural invasion or sometimes a technological colonization. Thus, we need to support those who are interested in improving the Quality of Life (QOL) of people in developing countries by cooperating the user research and designing something new that will contribute to the QOL of people living there, as well as conducting the conventional localization.

\section{Workshop Organization and Duration, Target Audience, and Expected Outcomes}

This one-day workshop will address the goals above through three activities: (a) workshop participants will together compile a list of examples of indigenous HCI problems, (b) participants will present short papers on their own current research; (c) group discussion will address specific issues. The target audience is HCI researchers and practitioners who have experience with working with culture and HCI, e.g., participants in IWIPS, ICIC, IFIP TC 13 SIG on Interaction Design and International Development, and more. The expected outcome of the workshop is a) network building among the participants, b) a shortlist of papers that can be basis for a proposal for a special issue of the UAIS journal, and c) identify opportunities to develop a funded network or research proposal.

\section{References}

1. Suchman, L.: Located accountabilities in technology production. Scandinavian Journal of Information Systems 14(2), 7 (2002)

2. Button, G., Dourish, P.: Technomethodology: paradoxes and possibilities. In: CHI 1996, pp. 19-26. ACM, New York (1996)

3. Hofstede, G.: Culture's Consequences: Comparing Values, Behaviors, Institutions, and Organizations Across Nations. Sage Publications, Thousand Oaks (2001)

4. Kaptelinin, V., Nardi, B.: Acting with technology: Activity theory and interaction design. MIT Press, Cambridge (2006)

5. Kurosu, M., Kobayashi, T., Yoshitake, R., Takahashi, H., Urokohara, H., Sato, D.: Trend in Usability Research and Activities in Japan. International Journal of Human-Computer Interaction 17(1), 103-124 (2004) 